\title{
Orofacial Granulomatosis: Clinical Signs of Different Pathologies
}

\author{
Giuseppe Troiano $^{\mathrm{a}}$ Mario Dioguardi $^{\mathrm{a}}$ Giovanni Giannatempo ${ }^{\mathrm{a}}$ \\ Luigi Laino $^{a}$ Nunzio Francesco Testa ${ }^{c}$ Roberto Cocchi ${ }^{b}$ Alfredo De Lillo ${ }^{a}$ \\ Lorenzo Lo Muzio ${ }^{a}$ \\ a Department of Clinical and Experimental Medicine, Foggia University, and ${ }^{b}$ Unit of Maxillofacial Surgery, \\ Casa Sollievo della Sofferenza, S. Giovanni Rotondo, Foggia, and ' Department of Translational Medicine, \\ Bari University, Bari, Italy
}

\section{Key Words}

Orofacial granulomatosis - Inflammatory bowel disease .

Crohn's disease - Sarcoidosis - Granulomatosis with

polyangiitis · Melkersson-Rosenthal syndrome

\begin{abstract}
Orofacial granulomatosis (OFG) is an uncommon disease characterized by persistent or recurrent soft tissue enlargement, oral ulceration and a variety of other orofacial features. It could be an oral manifestation of a systemic disease. For a correct differential diagnosis, local and systemic conditions characterized by granulomatous inflammation should be excluded using appropriate clinical and laboratory investigations. In fact, the diagnosis of OFG may be confirmed only by histopathological identification of noncaseating granulomas. The literature from 1943 to 2014 was reviewed with emphasis on the etiology of OFG and on clinical manifestations of systemic pathologies associated with OFG. The precise cause of OFG is still unknown, although several theories have been suggested, such as infection, hereditary factors and allergy. OFG is a disease that has a wide spectrum of presentation, which may include the oral manifestation of a systemic condition such as Crohn's disease, sarcoidosis, granulomatosis with polyangiitis and Melkersson-Rosenthal syndrome.
\end{abstract}

(c) 2015 S. Karger AG, Basel

\section{KARGER 125}

E-Mail karger@karger.com www.karger.com/mpp

\section{(C) 2015 S. Karger AG, Basel}

1011-7571/15/0242-0117\$39.50/0

Karger

Open access

This is an Open Access article licensed under the terms of the Creative Commons Attribution-NonCommercial 3.0 Unported license (CC BY-NC) (www.karger.com/OA-license), applicable to the online version of the article only. Distribution permitted for non-commercial purposes only.

\section{Introduction}

Orofacial granulomatosis (OFG) includes a group of diseases characterized by the presence of noncaseating granulomatous inflammation affecting the soft tissues of the oral and maxillofacial region [1]. OFG may be the oral manifestation of a systemic condition such as inflammatory bowel disease (IBD), sarcoidosis, granulomatosis with polyangiitis (GPA) and Melkersson-Rosenthal syndrome (MRS) [2-4]. These pathologies are part of a differential diagnosis of other granulomatous diseases, such as tuberculosis, leprosy, systemic fungal infections and foreign body reactions [5]. The diagnosis of OFG is made by histopathological identification of noncaseating granulomas. Local and systemic conditions characterized by granulomatous inflammation should be excluded by appropriate clinical and laboratory investigations [6]. This review focuses on the oral clinical aspects and the etiology of OFG.

A review of the literature was carried out using the computerized database 'PubMed Medline' without specifying the year of publication. Inclusion criteria were papers searched by key word or titles using the following combinations: orofacial granulomatosis, oral manifestations of inflammatory bowel disease, oral sarcoidosis, oral manifestations of granulomatosis with polyangiitis and MelkerssonRosenthal syndrome. The abstracts of the articles were

Prof. Lorenzo Lo Muzio

Department of Clinical and Experimental Medicine

Foggia University

Via Rovelli, 50, IT-71122 Foggia (Italy)

E-Mail llomuzio@tin.it 
reviewed in order to exclude irrelevant studies, and clinical opinion papers were excluded. Attention was paid to other articles that did not appear in the main search, by the reference lists of key articles in the field. Only English abstracts were included in the study. Following the preliminary identification of 123 articles, only 24 were identified as meeting the inclusion criteria. The following oral manifestations were observed: IBD, sarcoidosis, GPA and MRS.

\section{Inflammatory Bowel Disease}

IBD comprises two chronic clinical entities: Crohn's disease (CD) and ulcerative colitis (UC), both immunologically based [7]. CD can cause transmural inflammation and affect any part of the gastrointestinal tract (most commonly, the terminal ileum or the perianal region) in a discontinuous type. CD is commonly associated with complications such as abscesses, fistulas and strictures, but UC is characterized by mucosal inflammation and limited to the colon [8-10]. The key factors responsible for IBD include genetic components, environmental factors, microbial flora and immune responses. IBD arises from an extremely complex interaction among genetic and environmental factors, dysregulated immune responses and alterations in the microbiota, but none of these factors alone is likely to cause the disease [11]. Bowel symptoms are predominant, but extraintestinal complications may occur in these pathologies, including the involvement of the oral cavity. CD and UC are commonly associated with abdominal pain, diarrhea, rectal bleeding, weight loss and signs of malnutrition [12]. Approximately one third of IBD patients develop extraintestinal manifestations in the course of their disease [13]. The most common extraintestinal manifestations involve the joints, skin, eyes and biliary tract [14].

The association between OFG and IBD was especially noted in CD rather than in other IBD events. Oral manifestations have a prevalence ranging from 20 to $50 \%$, and initial presenting signs occur in $5-10 \%$ of affected patients (fig. 1a). Lesions are usually more severe during active disease, but up to $30 \%$ of patients may continue to manifest oral lesions despite disease control [15]. The most common intraoral site seems to be the lips. The oral manifestations of $\mathrm{CD}$ can be divided into specific and nonspecific lesions, based on the presence/absence of granuloma during the histopathological examination (table 1). Specific lesions are less common than nonspecific ones and, at histopathological examination, present typical granuloma characteristics [16].
Table 1. Specific and nonspecific oral lesions of CD

\begin{tabular}{ll}
\hline Specific lesions & Nonspecific lesions \\
\hline Indurated tag-like lesions & Aphthous stomatitis \\
Cobblestoning & Pyostomatitis vegetans \\
Mucogingivitis & Angular cheilitis \\
Lip swelling with vertical fissures & Glossitis \\
Deep linear ulceration & Persistent submandibular \\
& lymphadenopathy \\
\hline
\end{tabular}

Indurated tag-like lesions are white reticular tags referred to as mucosal tags, epithelial tags or folds. Another kind of presentation is called 'cobblestoning', which means fissured swollen buccal mucosa with corrugation and hyperplastic behavior of the mucosa. The lesions often appear as mucosal-colored papules that produce firm plaques on the buccal mucosa and palate. These lesions are pathognomonic for $\mathrm{CD}$ but are not associated with intestinal CD activity $[17,18]$. Another oral involvement common to CD is a particular form of mucogingivits. The whole gingiva, up to the mucogingival line, might be involved. The gingiva may become edematous, granular and hyperplastic with or without ulceration $[19,20]$. Other specific lesions are deep swelling with vertical fissures and deep linear ulceration. These lesions could cause pain and are a discomfort to the patients; they are not associated with intestinal activity. Nonspecific lesions are not pathognomonic of CD (table 1).

\section{Sarcoidosis}

Sarcoidosis is a systemic granulomatous disease of unknown cause affecting mostly young and middle-aged adults. The highest annual incidence of sarcoidosis has been observed in northern European countries (5-40 cases per 100,000 people) [21]. This pathology affects multiple organs, especially the lungs, lymph nodes, skin and eyes. Some studies confirm a genetic predisposition to sarcoidosis and present evidence for the allelic variation at the HLA-DRB1 locus as a major cause [22]. In the maxillofacial region, the salivary glands are often involved, and this may cause xerostomia or bilateral parotid swelling [23-25]. The involvement of the oral cavity seems to be rare with an unknown prevalence. Oral sarcoidosis usually appears in patients with chronic multisystemic sarcoidosis and presents as first manifestation of the disease in one third to almost two thirds of patients [26]. In 1943, Poe [27] reported the first documented case of oral sarcoidosis; since then, 
Fig. 1. a Intraoral manifestation of CD. b Oral sarcoidosis: intraoral granulomatosis. c Histological sample of oral tissues in a patient with sarcoidosis. d Radiological aspect of the lung in a patient with sarcoidosis. e Strawberry-like gingivitis in a patient with GPA. $\mathbf{f} \mathrm{MC}$ in a patient with MRS.
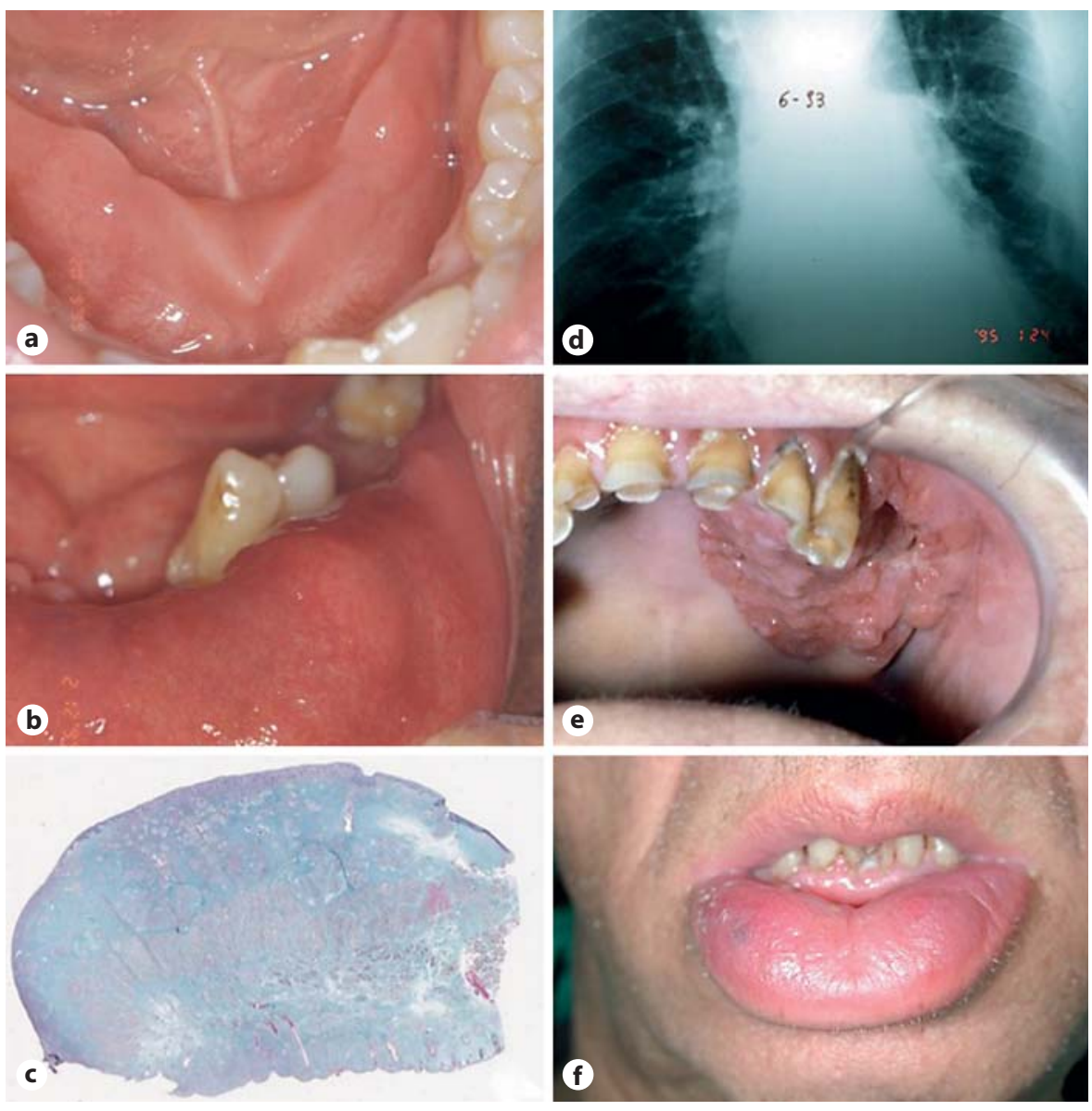

there have been only 68 well-documented cases of isolated oral sarcoidosis reported in the English literature. These lesions were localized swellings or nodules $(n=34$; fig. $1 b)$, ulcers $(n=4)$, swelling with multiple ulcers $(n=2)$, gingivitis $(\mathrm{n}=3)$, gingival hyperplasia $(\mathrm{n}=3)$ and gingival recession $(\mathrm{n}=1)$. In 24 cases, these oral lesions were the first manifestation of systemic sarcoidosis [28, 29].

Only some cases of oral sarcoidosis require treatment, because in nearly $60 \%$ of patients, the symptoms resolve spontaneously within 2 years. When lesions are localized, surgical excision is commonly employed, allowing both histopathological confirmation of sarcoidosis and treatment with systemic corticosteroids (fig. 1c, d) [30].

\section{Granulomatosis with Polyangiitis}

GPA is a systemic inflammatory disease characterized by necrotizing granulomatous inflammation and small-vessel vasculitis of the upper and lower respira- tory tract and kidneys; however, other organs or tissues may be affected [31]. The world prevalence of GPA seems to be 23.7-156.5 cases per million [32], with an estimated annual incidence of 3.0-14.4 cases per million [33]. Oral manifestations of GPA range from 6 to $13 \%$ of patients, and the mouth may be the initial site of clinical presentation in $5-6 \%$ of cases $[34,35]$. The most common oral manifestations of GPA are strawberry-like gingivitis, erythema, osteonecrosis of the palate, desquamation and nodular masses of the lips, petechiae and ulceration. Particularly strawberry-like gingivitis or hyperplastic gingival lesions are suggested to be a characteristic sign of GPA (fig. 1e) [36-38]. This sign consists of reddish-purple exophytic gingival swellings with petechial hemorrhages, thus similar to strawberries. This lesion can remain localized in the oral cavity for unusually long periods of time before multiorgan involvement occurs [39]. 


\section{Melkersson-Rosenthal Syndrome}

MRS is a rare, noncaseating granulomatous disease consisting of persistent or recurrent orofacial edema, relapsing peripheral facial paralysis and a fissured tongue [40]. Similar oral granulomatous reactions that are not associated with other detectable systemic diseases or foreign bodies may be present in Miescher's cheilitis (MC). MC is a term used when lip swelling is the unique clinical sign (fig. 1f) [41]. MRS is also associated with some other syndromes, including trigeminal neuralgia, paresthesias, ocular palsies, blepharospasm, epiphora, keratitis, psychotic episodes, uveitis and migraine $[42,43]$. MC may occur without other signs of the disease but may also be part of MRS; it could be a manifestation of CD and, rarely, of sarcoidosis. It is still debated whether MC should be considered as one of the extraintestinal manifestations of $\mathrm{CD}$ which can optionally be preceded by a few years of digestive problems or as a different disease entity. Van der Waal et al. [44], in a study of $13 \mathrm{MC}$ cases, reported the appearance of gastrointestinal lesions of CD in 2 cases after a follow-up period of 2 years. This association has been supported by the results of another study [45]. Because of this association, in patients with MC, Pittock [46] recommended a screening for gastrointestinal lesions especially in children and young patients. The value of a systematic research of gastrointestinal lesions of MC has been reinforced by the fact that about one third of patients with OFG manifestations may have asymptomatic intestinal inflammatory lesions [44]. The treatment of MRS is controversial. Various modes of treatment have been proposed, including nonsteroidal antiinflammatory drugs, antibiotics, antihistamines, lymecycline, systemic and intralesional corticosteroids and methotrexate [47-49]. The fissured tongue is congenital and is usually asymptomatic and benign and does not require any treatment [45].

\section{Etiology of OFG}

Introduced by Wiesenfeld et al. [2] in 1985, the term 'etiology of orofacial granulomatosis' is used to describe diseases which have a variety of clinical presentations but also a similar histopathological picture to nonspecific, noncaseating granulomatous inflammation. OFG is either considered a different pathology or a manifestation of systemic disease $[2,50]$. The precise cause of OFG is still unknown, although several theories have been suggested including infection, hereditary factors and allergy.
A genetic predisposition has been evaluated in different studies; some of these suggested the occurrence of the disease in families and a frequent expression of some HLA antigens among the patients compared with the normal population, possibly of autosomal dominant transmission with incomplete penetration and translocation at chromosome 9p11 [51, 52]. It seems that consistent HLA genotypes are evident in patients with OFG, in particular A2/3, B7 and DR 2/3/4. These genotypes are known to occur in haplotype association. More studies are needed to validate these claims [53]. The possible role of infection in the pathogenesis of OFG has been suggested because of its involvement in $\mathrm{CD}$ and sarcoidosis [54]. Most studies have focused on Mycobacterium tuberculosis, M. paratuberculosis, Staphylococcus aureus and Borrelia burgdorferi [50-53]. Apaydin et al. [55] had used a ribosomal RNA amplification-based Gen-Probe amplified $M$. tuberculosis direct test to determine the presence of the $M$. tuberculosis complex in skin biopsy specimens from 5 patients with MRS and 1 patient with OFG. Three (all belonging to the MRS samples) of the 6 patients were positive; 1 of the positive specimens also showed positive Ziehl-Neelsen staining. M. paratuberculosis was investigated by Riggio et al. [56] in 1997 using polymerase chain reaction on 37 samples (30 OFG and 7 $\mathrm{CD})$. The results of this study suggested that in this patient group, M. paratuberculosis did not appear to be associated with OFG or the oral lesions of CD [56]. S. aureus had been detected in mouths of 4 patients cultured using the oral rinse technique. It could represent a potential cause of panstomatitis in patients with OFG [57]. Some Chinese studies that evaluated the role of spirochetes in the etiology of OFG using Warthin-Starry special stain detected antibodies against B. burgdorferi in up to $82 \%$ of patients with cheilitis granulomatosa and MRS $[58,59]$. However, Muellegger et al. [60] who examined a retrospective study of 12 patients with OFG using polymerase chain reaction, ELISA and immunoblot test suggested that $B$. burgdorferi was not involved in the pathogenesis of OFG [60]. Hence, more studies are needed to clarify the role of this spirochete as an etiological factor of OFG.

Allergy in the form of hay fever, atopic eczema or asthma has been reported as being more prevalent, affecting $12-60 \%$ of OFG patients compared to $15 \%$ in the general population $[61,62]$. Many diseases affecting the orofacial region have an allergic basis, and the role of dietary antigens, in particular in the etiopathogenesis of OFG, seems to be compelling [63]. Examples include cinnamon and benzoates which were positive on patch 
tests in patients with a variety of oral mucosal diseases including OFG [64]. A study of 48 patients with OFG who were patch tested for their reaction to common food additives showed that 10 had a positive skin reaction and 7 showed improvement in their OFG with an elimination diet $[62,65]$. Occasionally, even delayed hypersensitivity to dental materials had been implicated in the etiology of OFG. Granulomatous reactions have been found in amalgam tattoos and in subcutaneous deposits of inorganic mercury $[66,67]$. One study assessing the utility of the cutaneous patch test showed that patients with OFG had more reactions to food additives, especially benzoic acid and chocolate, than other disease co- horts or controls [64]. However, there is no conclusive evidence to support a role for allergy as the cause of OFG.

\section{Conclusion}

OFG is a disease with a wide spectrum of presentations. OFG may be the oral manifestation of a systemic condition, such as IBD, sarcoidosis, GPA and MRS. The precise cause of OFG is still unknown. However, several theories have suggested an important role for infection, hereditary factors and allergy.

\section{References}

1 Sciubba JJ, Said-Al-Naief N: Orofacial granulomatosis: presentation, pathology and management of 13 cases. J Oral Pathol Med 2003; $32: 576-585$

2 Wiesenfeld D, Ferguson MM, Mitchell DN, et al: Oro-facial granulomatosis - a clinical and pathological analysis. Q J Med 1985;54:101113.

3 Girlich C, Bogenrieder T, Palitzsch KD, et al: Orofacial granulomatosis as initial manifestation of Crohn's disease: a report of two cases. Eur J Gastroenterol Hepatol 2002;14:873876.

4 Mignogna MD, Fedele S, Lo Russo L, et al: Orofacial granulomatosis with gingival onset. J Clin Periodontol 2001;28:692-696.

5 Alawi F: Granulomatous diseases of the oral tissues: differential diagnosis and update. Dent Clin North Am 2005;49:203-221, x.

6 Rana AP: Orofacial granulomatosis: a case report with review of literature. J Indian Soc Periodontol 2012;16:469-474

7 Trost LB, McDonnell JK: Important cutaneous manifestations of inflammatory bowel disease. Postgrad Med J 2005;81:580-585.

8 Abraham C, Cho JH: Inflammatory bowel disease. N Engl J Med 2009;361:2066-2078.

9 Katsinelos P, Tziomalos K, Fasoulas K, et al: Can capsule endoscopy be used as a diagnostic tool in the evaluation of nonbleeding indications in daily clinical practice? A prospective study. Med Princ Pract 2011;20:362-367.

10 Spencer AJ: An evidence-based approach to the prevention of oral diseases. Med Princ Pract 2003;12(suppl 1):3-11.

11 Zhang YZ, Li YY: Inflammatory bowel disease: pathogenesis. World J Gastroenterol 2014;20:91-99.

12 Lourenco SV, Hussein TP, Bologna SB, et al: Oral manifestations of inflammatory bowel disease: a review based on the observation of six cases. J Eur Acad Dermatol Venereol 2010 24:204-207.

Orofacial Granulomatosis
13 Armond MC, Carlos RG, Pazzini CA, et al: Crohn's disease: clinical manifestations of orthodontic interest. Am J Orthod Dentofacial Orthop 2011;139:704-707.

14 Levine JS, Burakoff R: Extraintestinal manifestations of inflammatory bowel disease. Gastroenterol Hepatol (NY) 2011;7:235-241.

15 Lankarani KB, Sivandzadeh GR, Hassanpour $S$ : Oral manifestation in inflammatory bowel disease: a review. World J Gastroenterol 2013; 19:8571-8579.

16 Plauth M, Jenss H, Meyle J: Oral manifestations of Crohn's disease. An analysis of 79 cases. J Clin Gastroenterol 1991;13:29-37.

17 Harty S, Fleming P, Rowland M, et al: A prospective study of the oral manifestations of Crohn's disease. Clin Gastroenterol Hepatol 2005;3:886-891.

18 Williams AJ, Wray D, Ferguson A: The clinical entity of orofacial Crohn's disease. Q J Med 1991;79:451-458.

19 Harikishan G, Reddy NR, Prasad H, et al: Oral Crohn's disease without intestinal manifestations. J Pharm Bioallied Sci 2012;4:S431S434.

20 Katz J, Shenkman A, Stavropoulos F, et al: Oral signs and symptoms in relation to disease activity and site of involvement in patients with inflammatory bowel disease. Oral Dis 2003;9:34-40.

21 Pietinalho A, Hiraga Y, Hosoda Y, et al: The frequency of sarcoidosis in Finland and Hokkaido, Japan. A comparative epidemiological study. Sarcoidosis 1995;12:61-67.

22 Rossman MD, Thompson B, Frederick M, et al: HLA-DRB1*1101: a significant risk factor for sarcoidosis in blacks and whites. Am J Hum Genet 2003;73:720-735.

23 Clayman L, MacLennan M, Dolan RL: Nonpainful swelling of the palate and loosening of the maxillary incisors. J Oral Maxillofac Surg 1998;56:1327-1335.

24 Iannuzzi MC, Rybicki BA, Teirstein AS: Sarcoidosis. N Engl J Med 2007;357:2153-2165.
25 Abul A, Onadeko BO, Khadadah ME, et al: Clinical patterns of diffuse parenchymal lung disease in Kuwait: a prospective study. Med Princ Pract 2004;13:78-83.

26 Jackowski J, Dragisic D, Arnold G, et al: Primary oral sarcoidosis preceding Lofgren's syndrome. Oral Surg Oral Med Oral Pathol Oral Radiol Endod 2005; 100:183-185.

27 Poe DL: Sarcoidosis of the jaw - a new disease of the mandible. Am J Orthod Dentofacial Orthop 1943;29:52-56.

28 Suresh L, Radfar L: Oral sarcoidosis: a review of literature. Oral Dis 2005;11:138-145.

29 Bouaziz A, Le Scanff J, Chapelon-Abric C, et al: Oral involvement in sarcoidosis: Report of 12 cases. QJM 2012;105:755-767.

30 Kasamatsu A, Kanazawa H, Watanabe T, et al: Oral sarcoidosis: report of a case and review of literature. J Oral Maxillofac Surg 2007;65: 1256-1259.

31 Almouhawis HA, Leao JC, Fedele S, et al: Wegener's granulomatosis: a review of clinical features and an update in diagnosis and treatment. J Oral Pathol Med 2013;42:507-516.

32 Abdou NI, Kullman GJ, Hoffman GS, et al Wegener's granulomatosis: survey of $701 \mathrm{pa}-$ tients in North America. Changes in outcome in the 1990s. J Rheumatol 2002;29:309-316.

33 Reinhold-Keller E, Herlyn K, Wagner-Bastmeyer $\mathrm{R}$, et al: Stable incidence of primary systemic vasculitides over five years: results from the German vasculitis register. Arthritis Rheum 2005;53:93-99.

34 Ponniah I, Shaheen A, Shankar KA, et al: Wegener's granulomatosis: The current understanding. Oral Surg Oral Med Oral Pathol Oral Radiol Endod 2005;100:265-270.

35 Patten SF, Tomecki KJ: Wegener's granulomatosis: cutaneous and oral mucosal disease. J Am Acad Dermatol 1993;28:710-718.

36 Bachmeyer C, Petitjean B, Testart F, et al: Lingual necrosis as the presenting sign of Wegener's granulomatosis. Clin Exp Dermatol 2006; $31: 321-322$. 
37 Bhatt V, Hall TJ: Strawberry gingival enlargement as only manifestation of Wegener's granulomatosis. Br J Oral Maxillofac Surg 2009;47:500.

38 Bulbul Y, Ozlu T, Oztuna F: Wegener's granulomatosis with parotid gland involvement and pneumothorax. Med Princ Pract 2003;12: 133-137.

39 Stewart C, Cohen D, Bhattacharyya I, et al: Oral manifestations of Wegener's granulomatosis: a report of three cases and a literature review. J Am Dent Assoc 2007;138:338-348, quiz 396, 398.

40 Zimmer WM, Rogers RS 3rd, Reeve CM, et al: Orofacial manifestations of MelkerssonRosenthal syndrome. A study of 42 patients and review of 220 cases from the literature. Oral Surg Oral Med Oral Pathol 1992;74:610619.

41 Scully C, Langdon J, Evans J: Marathon of eponyms: 13 Melkersson-Rosenthal syndrome. Oral Dis 2010;16:707-708.

42 Aluclu MU, Keklikci U, Guzel A, et al: Melkersson-Rosenthal syndrome with partial oculomotor nerve palsy. Ann Saudi Med 2008;28: 135-137.

43 Hazey MA, Van Norman AJ, Armistead DL: Melkersson-Rosenthal syndrome with migraine-like headaches treated with minocycline: a case report and review of the literature. W V Med J 2009;105:15-17.

44 van der Waal RI, Schulten EA, van der Meij $\mathrm{EH}$, et al: Cheilitis granulomatosa: overview of 13 patients with long-term follow-up - results of management. Int J Dermatol 2002;41: 225-229.

45 Liu R, Yu S: Melkersson-Rosenthal syndrome: a review of seven patients. J Clin Neurosci 2013;20:993-995.

46 Pittock S, Drumm B, Fleming P, et al: The oral cavity in Crohn's disease. J Pediatr 2001; 138 : 767-771.
47 Bacci C, Valente ML: Successful treatment of cheilitis granulomatosa with intralesional injection of triamcinolone. J Eur Acad Dermatol Venereol 2010;24:363-364.

48 Tonkovic-Capin V, Galbraith SS, Rogers RS, et al: Cutaneous Crohn's disease mimicking Melkersson-Rosenthal syndrome: treatment with methotrexate. J Eur Acad Dermatol Venereol 2006;20:449-452.

49 Wickramasinghe N, Gunasekara CN, Fernando WS, et al: Vulvitis granulomatosa, Melkersson-Rosenthal syndrome, and Crohn's disease: dramatic response to infliximab therapy. Int J Dermatol 2012;51:966-968.

50 Sanderson J, Nunes C, Escudier M, et al: Orofacial granulomatosis: Crohn's disease or a new inflammatory bowel disease? Inflamm Bowel Dis 2005;11:840-846.

51 Goto A, Kume A, Aiba I, et al: A case of typical Melkersson-Rosenthal syndrome with possible autosomal dominant inheritance. Rinsho Shinkeigaku 1999;39:1020-1024.

52 Smeets E, Fryns JP, Van den Berghe H: Melkersson-Rosenthal syndrome and de novo autosomal $\mathrm{t}(9 ; 21)$ (p11;p11) translocation. Clin Genet 1994;45:323-324.

53 Gibson J, Wray D: Human leucocyte antigen typing in orofacial granulomatosis. Br J Dermatol 2000;143:1119-1121.

54 O’Neill RP, Penman RW: 'Sarcoidosis' complicated with nocardiosis. J Ir Med Assoc 1969;62:287-290.

55 Apaydin R, Bahadir S, Kaklikkaya N, et al: Possible role of mycobacterium tuberculosis complex in Melkersson-Rosenthal syndrome demonstrated with Gen-Probe amplified $M y$ cobacterium tuberculosis direct test. Australas J Dermatol 2004;45:94-99.

56 Riggio MP, Gibson J, Lennon A, et al: Search for Mycobacterium paratuberculosis DNA in orofacial granulomatosis and oral Crohn's disease tissue by polymerase chain reaction. Gut 1997;41:646-650.
57 Gibson J, Wray D, Bagg J: Oral staphylococcal mucositis: a new clinical entity in orofacial granulomatosis and Crohn's disease. Oral Surg Oral Med Oral Pathol Oral Radiol Endod 2000;89:171-176.

58 Liu HG: Spirochetes in the cheilits granulomatosa and sarcoidosis. Zhonghua Yi Xue Za Zhi 1993;73:142-144, 189-190.

59 Liu HG, Zheng LF, Xiao XZ: Relationship between Melkersson-Rosenthal syndrome and spirochetes infection. Zhonghua Yi Xue Za Zhi 1994;74:92-93, 127.

60 Muellegger RR, Weger W, Zoechling N, et al: Granulomatous cheilitis and Borrelia burgdorferi: polymerase chain reaction and serologic studies in a retrospective case series of 12 patients. Arch Dermatol 2000;136:15021506.

61 Armstrong DK, Burrows D: Orofacial granulomatosis. Int J Dermatol 1995;34:830-833.

62 Armstrong DK, Biagioni P, Lamey PJ, et al: Contact hypersensitivity in patients with orofacial granulomatosis. Am J Contact Dermat 1997;8:35-38.

63 Reed BE, Barrett AP, Katelaris C, et al: Orofacial sensitivity reactions and the role of dietary components. Case reports. Aust Dent J 1993;38:287-291.

64 Wray D, Rees SR, Gibson J, et al: The role of allergy in oral mucosal diseases. QJM 2000;93 507-511.

65 White A, Nunes C, Escudier M, et al: Improvement in orofacial granulomatosis on a cinnamon- and benzoate-free diet. Inflamm Bowel Dis 2006;12:508-514

66 Buchner A, Hansen LS: Amalgam pigmentation (amalgam tattoo) of the oral mucosa. A clinicopathologic study of 268 cases. Oral Surg Oral Med Oral Pathol 1980;49:139-147.

67 Lazarov A, Kidron D, Tulchinsky Z, et al Contact orofacial granulomatosis caused by delayed hypersensitivity to gold and mercury. J Am Acad Dermatol 2003;49:1117-1120. 